\title{
wingless refines its own expression domain on the Drosophila wing margin
}

Eric J. Rulifson, Craig A. Micchelli, Jeffrey D. Axelrod, Norbert Perrimon \& Seth S. Blair

Nature 384, 72-74 (1996)

BECAUSE of an error in the production process all the figures in this Letter were unclear and of poor quality. They are reproduced again below.

FIG. 1 a-i, Margin wg expression after loss of $w g$ or dsh functions. a, Diagram of late third instar wing disc. Box outlines margin region shown in the remaining figures, with axes and patterns of gene expression as marked (see text for details). AS-C, acheatescute complex expression; DN, dorsoventral. $b-e, w g^{t s}\left(w g^{t / L} / g^{c \times 4}\right)$ margins, stained for $\mathrm{Wg}$ protein $(b, c)$ or messenger RNA $(d, e)$ expression. $b$, $d$, At permissive temperature, protein and mRNA expression was nomal. $c$, e, After a $12 \mathrm{~h}$ shift to restrictive temperature, protein expression expanded to a region twice the nomal width (protein: 55/57 discs; mRNA: 17/19 discs). $f$, g, Hypomorphic wglacz clone on the margin, shown by the absence of anti-Myc staining $(f ;$ in $g$ and subsequent panels clones are marked by a dotted outline and ${ }^{*}$ ). Clones caused expansion of $w g-L a c Z$ expression (anti- $\beta$-gal; $72 / 97$, none showed loss). $h, i, d s h$ clones that intersected $(h)$ or sat immediately adjacent to $(i)$ the wg stripe elevated anti-Wg-staining cellautonomously ( $d s h^{75}$ : 15/17; $d s h^{26}$.
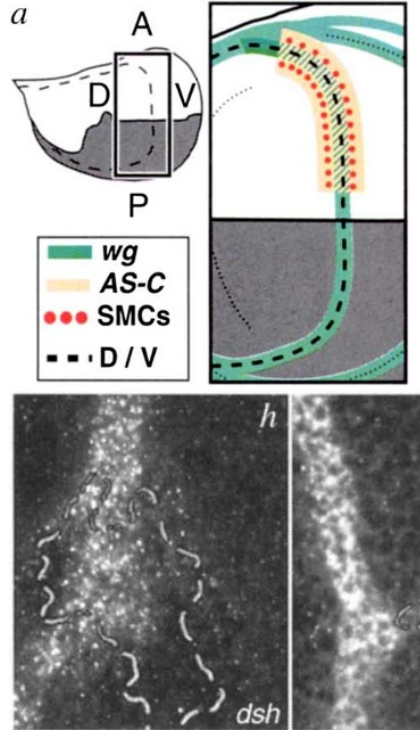
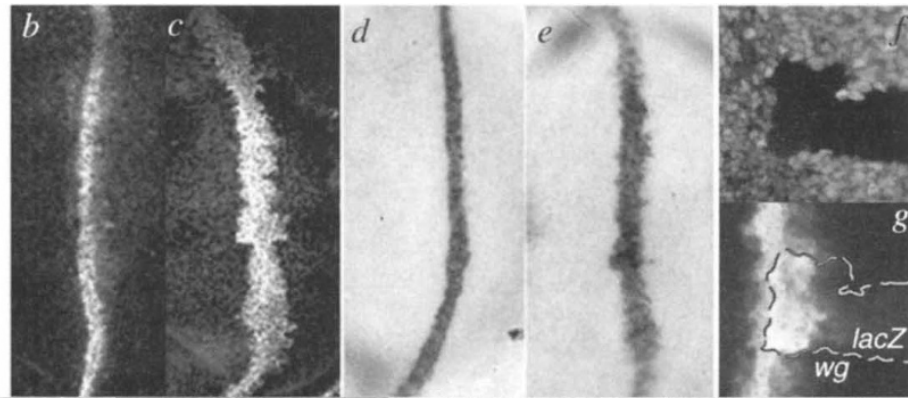

$12 / 12$ ). Similar effects were observed in anterior, posterior, dorsal and ventra clones. In some cases, it seemed that the normal wg stripe expanded or distorted to meet the ectopic anti-Wg staining within clones away from the margin (see $i ; 7$ clones). It is unclear whether ectopic wg expression is being induced in wild-type cells between the clone and the margin, or if cells at the margin are distorting or rearranging near the clone. $j, k$, Anti-Scute or Achaete staining in $w g$ and dsh clones. $j, w g^{-x / 4}$ clones that intersected the wg stripe showed non-autonomous loss of Sc (18/22 large clones), as did $5 / 7 w^{\text {Lacz }}$ clones (not shown). Sc was expressed at wild-type levels in wg mutant cells that were 1-2 cell diameters from the clone boundary, presumably in response to Wg secreted by wild-type cells. Most small clones, where all cells were
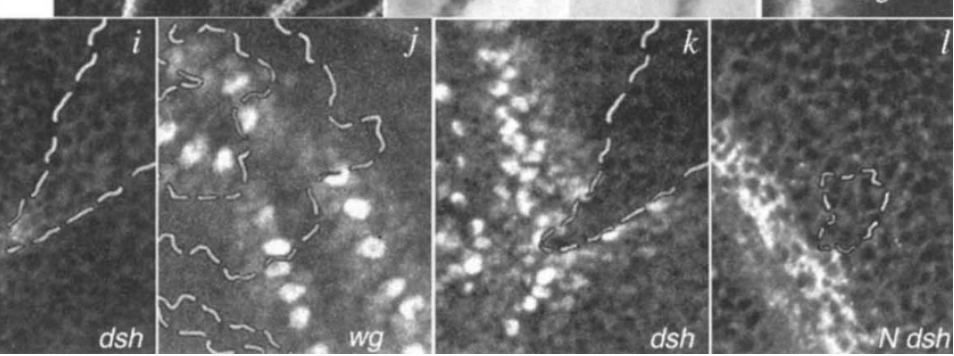

FIG. $2 d s h^{-}$clones induced ectopic bristles off the wing margin in a wg-dosage-dependent fashion. $a, d s h^{-}$clone in adult wing, marked with $y$ and $f^{36 a}$ (dotted outline), extending from the margin into the interior of the wing blade. The average distance from farthest bristle to margin was 4.5 cells ( \pm 1.0 , range $3-7, n=23$ ). b, dsh clone induced in a $w^{1622}$ heterozygote. The average distance for all clones observed was 2.71 ( \pm 0.73 , range $2-4$, $n=14)$. $c$, dsh mutant clone induced in the

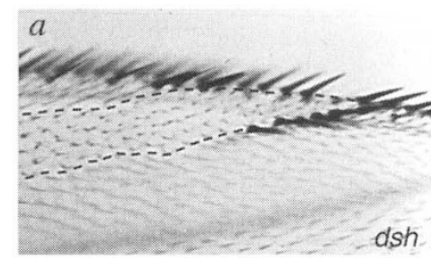

close to the boundary, showed normal Sc levels. $k, d s h^{-}$clones within the margin proneural region showed cell-autonomous loss of $s c$ expression $\left(d s h^{75}: 9 / 9 ; d s h^{26}\right.$ 15/15). dsh clones that intersected the wg stripe, and produced ectopic wg, also generated ectopic sc expression outside the normal proneural region in $d s h /+$ cells near the clone boundary (arrow; note wg expression in same clone in $i$ ). I, Margin wg expression after loss of Notch $(N)$ and dsh. Notch ${ }^{-1}$ sh $^{-}$clones that lay adjacent to $(I)$ or intersected the wg stripe (not shown) showed cell-autonomous loss of anti-Wg staining without expansion of wg expression (29/29 clones). Notch ${ }^{-}$dsh $^{-}$clones in the anterior also lose anti-Sc staining (E.J.R., C.A.M., M. Halevy and S.S.B., manu script in preparation)

presence of heat-shock promoter-wg. The average distance for all clones obsenved was 10 cells $(1.6$, range $8-12, n=5)$. hs-wg produces insufficient activity to generate ectopic bristles in a wild-type background (not shown). The clones in $b$ and $c$ are not marked with $f^{36 a}$, but are identified by the cell-autonomous tissue polarity defect caused by $d s h^{14}$ (results not shown). Unmarked $d s h^{-}$and Notch $^{-} d s h^{-}$clones were identified in

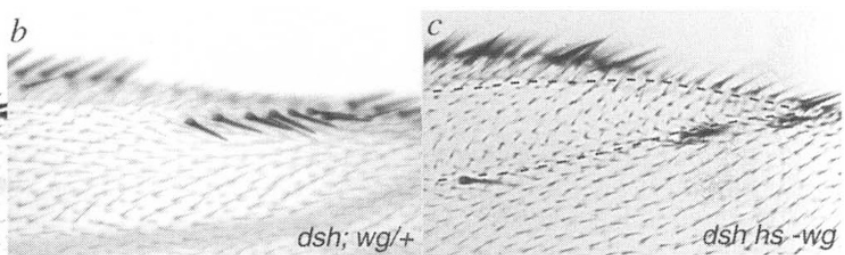

adult wings by the absence of normal margin bristles ${ }^{6,20}, 22 / 30$ dsh clones were accompanied by ectopic bristles distant from the margin, as compared with $5 / 40$ Notch $d s h$ clones. The bristles near Notch dsh clones were only rarely as contiguously arrayed or as distant from the margin as those near dsh clones. Regulation within the margin proneural regions may account for these occasional bristles (data not shown).
FIG. 3 Interactions between Notch and dsh. $a, b$, dsh overexpression in the posterior compartment, using UAS-dsh and en-GAL4, caused narrowing and occasional loss of margin anti-Wg staining $(b)$ when compared with anterior $(a)$. Dsh levels, as identified using anti-Dsh, were visibly higher in the posterior compartment (not shown). Both panels are from the same imaginal disc. c, Model of Wg self-refinement. Notch signalling activity is highest at the dorsoventral boundary, and levels above the threshold $\left(\Theta_{\mathrm{wg}}\right)$ initially trigger broad wg expression (above). Wg represses Notch (N) activity; wg expression is maintained only in cells nearest the dorsoventral boundary (below).
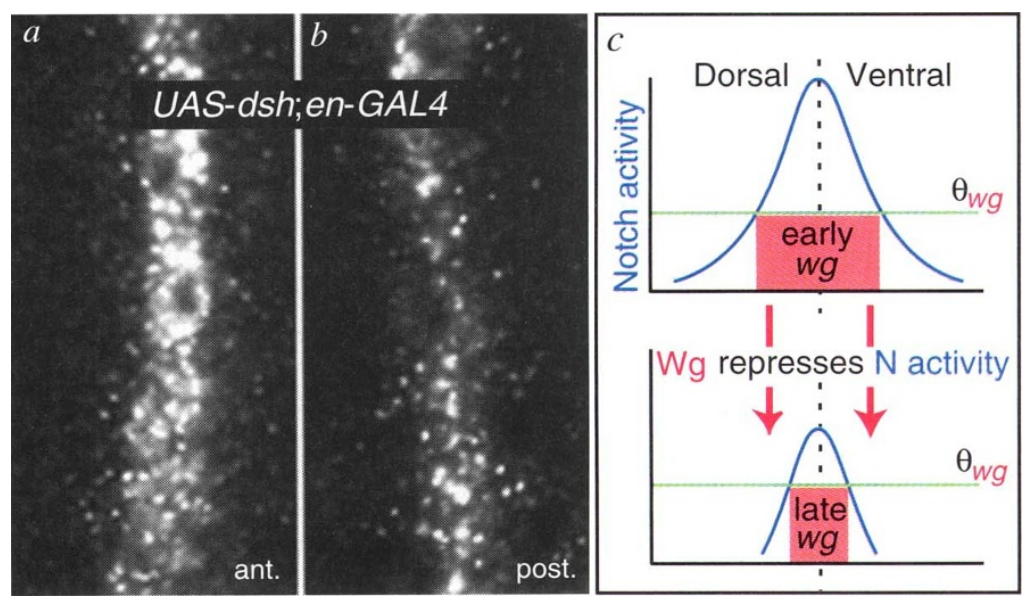